\title{
More Acquired Immunodeficiency Syndrome (AIDS) Dementia Complex in Japan?
}

It is well known that the clinical complex caused by infection with the retrovirus, human immunodeficiency virus-1 (HIV-1) is termed acquired immunodeficiency syndrome (AIDS). Although the primary site of infection in AIDS is thought to be leukocytes, a variety of neurological complications in AIDS has been described. In fact, HIV enters the central nervous system easily at the primary infection and may result in various stages of disorders; secondary opportunistic infections and neoplasmas, metabolic or nutritional derangements and HIV related-syndromes. According to Britton in Merritt's Textbook of Neurology, "neurological disorders are found in up to $70 \%$ of patients in clinical series of AIDS and in more than $80 \%$ of autopsied series. In 10 to $20 \%$, the neurological disorder is the first manifestation of AIDS" (1). This indicates the need for all neurologists worldwide to keep informed of the progress of AIDS research and medicine. Internists and neurologists in Japan are, of course, not exception.

Although the treatment protocols and means of management are not satisfactory at the moment, secondary opportunistic infections and neoplasmas are understandable manifestations of AIDS because HIVs destroy the most important protective apparatus. On the contrary, it is not easy to determine the mechanisms of the AIDS-related syndromes. In the early 1980s, sporadic and short descriptions of the disorders variously termed as subacute encephalitis or encephalopathy in AIDS patients have appeared in the literature. They are not caused by infection or neoplasma and are now recognized as AIDS dementia complex.

It is relevant here to cite a clear description of AIDS dementia complex from the above-mentioned Textbook of Neurology (1). "AIDS dementia complex is an insidiously progressive subcortical dementia. Early symptoms include apathy, social withdrawal, diminished libido, slow thinking, poor concentration, and forgetfulness. Psychiatric syndromes are sometimes profound and may be the first manifestation of HIV infection, expressed as psychosis, depression, or mania. Motor signs include slow movements, leg weakness, and gait ataxia. There may be headache, tremor, seizures, parkinsonian features, or frontal release signs. Although the disorder is usually progressive, some patients develop a static level of a static disability, and some improve in response to medical treatment for HIV or complicating disorders. Complete reversal is exceptional. When progressive, the disease culminates in akinetic mutism, an immobile bedridden state with global cognitive impairment and urinary incontinence". Pathological findings in such patients are not specific including microglial nodules, giant cells, focal perivascular demyelination and glio- sis, and neuronal cell loss especially in the frontal cortex, in the spinal cord, even in the peripheral nerves. Although HIV may be detected by in situ hybridization technique in these tissues, the actual pathogenesis of those clinical and pathological findings is uncertain. The exclusion of secondary infection, nutritional deficiency, or metabolic disorder is, of course, important but sometimes difficult.

The frequency of AIDS in general has increased tremendously throughout the world, especially in Africa and Asia. Although the absolute number of the patients with AIDS is still small in Japan, it is obviously increasing year by year. Responding to this general increase, patients with AIDS dementia complex are expected to increase. Therefore, it is important to consistently consider the possibility for a patient to have AIDS when his/her clinical manifestation includes dementia. In this respect, it is relevant that Arimura et al (2) collected 10 documented patients with AIDS dementia complex from the Japanese literature and reported an additional case of their own in this issue of the Journal, which is the first English language report from Japan on AIDS dementia complex. According to Arimura et al, 4 of the 11 patients were hemophiliacs who received intravenous administration of unheated factor VIII concentrate. This situation may be somewhat unique in Japan, as factor VIII agents were not made domestically but were imported from other countries. Whatever the reasons and situations were surrounding the decision to import the agents, outcome occurring in innocent hemophiliacs is obvious. Therefore, hemophiliacs received unheated factor VIII concentrate together with HIV should be econo-medically, and, needless to say, humanistically supported by the authoritative systems or agencys which gave permission to use the imported blood agents possibly contaminated with HIV. This is not a legal but rather an issue of morality.

Ichiro KanAZAWA, MD

Department of Neurology, University of Tokyo,

3-1, Hongo 7-chome, Bunkyo-ku, Tokyo 113

\section{References}

1) Britton CB. Acquired immunodeficiency syndrome (AIDS). in: Merritt's Textbook of Neurology, Rowland LP, Ed. Williams \& Wilkins, Baltimore, 1995, p.179.

2) Arimura H, Nakagawa M, Maruyama $Y$, et al. A hemophiliac with HIV1-associated dementia complex. Intern Med 34: 995, 1995. 